\title{
INFLOW RECONNECTION SOLUTIONS IN INCOMPRESSIBLE VISCO-RESISTIVE PLASMAS
}

\author{
I. J. D. Craig and Yuri E. Litvinenko \\ Department of Mathematics, University of Waikato, P. B. 3105, Hamilton, New Zealand \\ Received 2011 November 27; accepted 2012 January 14; published 2012 February 9
}

\begin{abstract}
The development of a visco-resistive length scale for the thickness of a reconnecting current sheet would have significant consequences for the physics of magnetic reconnection in solar flares. In this paper, planar magnetic reconnection in an incompressible visco-resistive plasma is investigated analytically and numerically. Relaxation simulations are performed in an "open" geometry that allows material to enter and exit the reconnection volume. Solutions of two types are identified depending on the strength of the external flow that drives the reconnection. For sufficiently strong flows separate resistive and viscous layers develop in the reconnection region. In this case merging rates are found to be largely independent of viscosity. However, when the flow is too weak to produce a localized current layer, an equilibrium lacking any small-scale structure is obtained. The central conclusion is that neither of these steady-state solutions provide evidence of a visco-resistive length scale.
\end{abstract}

Key words: magnetic reconnection - magnetohydrodynamics (MHD) - Sun: flares

\section{INTRODUCTION}

Magnetic reconnection is recognized as a key mechanism in the evolution of solar and stellar plasmas. In practice, the electrical resistivity of the plasma is often too small to determine the reconnection rate. In the solar corona, for example, the collisional resistivity corresponds to an inverse Lundquist number of order $\eta \simeq 10^{-14}$, a value that makes it difficult to obtain magnetic dissipation rates implied by observations of solar flares. Accordingly, recent reconnection research has focused on the search for extra physical processes that could speed up the reconnection rate, such as Hall effects or plasma turbulence (e.g., Cassak et al. 2006; Malakit et al. 2009; Litvinenko 2009, and references therein).

In the solar corona the dimensionless viscosity $v$ exceeds the dimensionless resistivity $\eta$ by many orders of magnitude. It is therefore reasonable to expect that plasma viscosity should strongly modify the reconnection process. Dimensional arguments for a visco-resistive Sweet-Parker current sheet (Park et al. 1983; Biskamp 1994) and analytical solutions for weakly perturbed magnetic $X$-points (Titov \& Priest 1997) predict that the speed of the reconnection outflow should decrease and the thickness of the current sheet should increase if $\eta \ll v \ll 1$. Specifically, the emergence of a visco-resistive length scale $(\eta v)^{1 / 4}$ for the current sheet thickness is predicted.

While the visco-resistive scale has been identified in reconnection simulations in closed line-tied $X$-points (Craig et al. $2005)$, it remains questionable whether the $(\eta \nu)^{1 / 4}$ scale can emerge in flux pile-up reconnection in an open geometry (Sonnerup \& Priest 1975). A preliminary study by Fabling \& Craig (1996) suggested that flux pile-up merging in an incompressible plasma should remain largely unaffected by viscous effects. The question is important in the context of modeling of energy release in solar flares. For typical coronal parameters we have $\eta^{1 / 2}<d_{i}<(\eta v)^{1 / 4}$ where $d_{i}$, the scale of the Hall effect, is the dimensionless ion skin depth based on the proton plasma frequency (see Section 2.1 below). Hence the visco-resistive scale for the current sheet thickness would be large enough to justify the use of fluid models to describe the structure of a flaring current sheet, whereas the resistive Sweet-Parker scale $\eta^{1 / 2}$ would strongly suggest the need for collisionless reconnection models.
Motivated by the conflicting results of the previous studies, in this paper we explore the influence of plasma viscosity on reconnection solutions by invoking an exact analytical reduction of the governing MHD equations. Specifically, we focus on generalizing the inflow solutions for reconnective annihilation (Craig \& Henton 1995) to the case of a viscous resistive plasma. In contrast to the steady-state treatment of Fabling \& Craig (1996), we examine the dynamic relaxation of the viscoresistive MHD system by formulating an initial value problem and investigating whether visco-resistive boundary layers appear in the quasi-steady solution. Moreover, when the viscoresistive layers do not emerge, we also explore the extent to which inviscid reconnection solutions (e.g., Craig \& Henton 1995) can approximate viscous flux pile-up merging.

The paper is organized as follows. In Section 2, we present the MHD system and discuss the analytical reduction that leads to reconnection solutions. The singular nature of the ideal system is discussed and it is pointed out that the system changes character when the external driving flow is no longer strong enough to localize the reconnecting magnetic field. Exact steady resistive solutions are then presented that reinforce the key role played by the external flow amplitude. In Section 3, the dynamic relaxation problem is formulated, and the resolution of singularities by resistive and viscous effects is investigated. It is shown that, depending on the strength of the flow, both localized current sheet solutions and extended diffuse solutions are possible. The impact of these findings is discussed in Section 4.

\section{FORMULATION OF THE PROBLEM AND IDEAL SINGULARITIES}

\subsection{Incompressible MHD Equations}

We consider the resistive MHD equations in an incompressible viscous plasma. These are written in dimensionless form by adopting reference values of the magnetic field $B_{0}$, plasma density $\rho_{0}$, and length $L$. Velocities are measured in units of the Alfvén speed $v_{A}=B_{0} / \sqrt{4 \pi \rho_{0}}$. A typical coronal number density $10^{9} \mathrm{~cm}^{-3}$ and $B_{0}=10^{2} \mathrm{G}$ give $v_{A} \simeq 10^{9} \mathrm{~cm} \mathrm{~s}^{-1}$. Times are scaled according to $t_{A}=L / v_{A}$ where $L=10^{9.5} \mathrm{~cm}$.

The dimensionless momentum and induction equations are given by

$$
\partial_{t} \mathbf{v}+(\mathbf{v} \cdot \nabla) \mathbf{v}=-\nabla p+(\nabla \times \mathbf{B}) \times \mathbf{B}+v \nabla^{2} \mathbf{v},
$$




$$
\partial_{t} \mathbf{B}=\nabla \times(\mathbf{v} \times \mathbf{B})+\eta \nabla^{2} \mathbf{B},
$$

where the $\mathbf{v}$ and $\mathbf{B}$ fields satisfy

$$
\nabla \cdot \mathbf{v}=0, \quad \nabla \cdot \mathbf{B}=0 .
$$

The system is controlled by two dimensionless parameters $v$ and $\eta$ which derive from collisional forms for the scalar viscosity and resistivity, normalized by $v_{A} L$ and $4 \pi v_{A} L / c^{2}$, respectively. For coronal applications we should take $v \simeq 10^{-4}$ and $\eta \simeq 10^{-14}$. In terms of the normalized ion skin depth $d_{i} \simeq 10^{-6.5}$ the ordering is $\eta^{1 / 2}<d_{i}<(\eta v)^{1 / 4}$, which suggests that resistive and Hall effects may be inhibited by the emergence of visco-resistive scales. Some caution must be exercised, however, in interpreting results based on a classical viscosity, as opposed to a more general anisotropic viscous stress tensor (Braginskii 1965; Hollweg 1986). Although the scalar form is strictly justified only in the vicinity of a magnetic neutral line (Craig \& Litvinenko 2005), the classical form is typically used in theoretical studies of visco-resistive reconnection (Titov \& Priest 1997; cf. Litvinenko 2005).

\subsection{Analytic Reduction}

A wide variety of reconnection solutions can be constructed using the forms

$$
\mathbf{v}=\alpha \mathbf{P}(\mathbf{x})+\mathbf{u}(\mathbf{x}, t), \quad \mathbf{B}=\beta \mathbf{P}(\mathbf{x})+\mathbf{b}(\mathbf{x}, t) .
$$

Here, $\alpha$ and $\beta$ are positive constants of order unity, $\mathbf{P}$ is a largescale background field and $\mathbf{u}$ and $\mathbf{b}$ are dynamic reconnection fields. Solutions are constructed by assuming reconnection fields of reduced dimensionality based on "fan" and "spine" forms for merging in two or three dimensions.

In the present application we consider only the simplest system, associated with a two-dimensional null $\mathbf{P}=(-x, y)$, namely,

$$
\mathbf{v}=-\alpha x \hat{\mathbf{x}}+(\alpha y+u(x, t)) \hat{\mathbf{y}}, \quad \mathbf{B}=\beta x \hat{\mathbf{x}}+(-\beta y+b(x, t)) \hat{\mathbf{y}}
$$

In this case $\alpha \geqslant 0$ defines the strength of a global stagnation point flow that washes curved field lines $(\beta>0)$ toward a merging region centered on the origin (Craig \& Henton 1995). We refer to the system as "flow dominated" when $\alpha>\beta$.

We are interested in determining reconnection fields $u$ and $b$ given plausible conditions on the boundary surfaces $x= \pm 1$. For reconnection fields that are odd functions of $x$, we have

$$
\begin{gathered}
u_{t}-\alpha x u_{x}+\alpha u=\beta x b_{x}-\beta b+\nu u_{x x}, \\
b_{t}-\alpha(x b)_{x}=\eta b_{x x}+\beta(x u)_{x} .
\end{gathered}
$$

Our aim is to explore this system using a combination of analytic and numerical techniques based on arbitrary initial conditions. However, the numerical relaxation is most conveniently performed using stream and flux function representations of the fields. The relaxation can then be parameterized by the flux transfer rate $E$-a rate that is both uniform and constant in steady two-dimensional reconnection solutions.

\subsection{The Ideal MHD System}

In the absence of dissipation the system of Equations (6) and (7) can be expected to develop singularities. Since the location and strength of these singularities can provide insight into the development of viscous and resistive boundary layers in the solution, it is instructive to consider the properties of the ideal system.

Setting $v=\eta=0$ and introducing the variables

$$
\tau=t, \quad s=\ln x+\alpha t,
$$

system (6) and (7) can be reduced to a Klein-Gordon equation for $b$, namely,

$$
b_{\tau \tau}-\beta^{2} b_{s s}=\left(\alpha^{2}-\beta^{2}\right) b .
$$

This equation is valid in fact for any linear combination of the fields $u$ and $b$. A formal solution of the Klein-Gordon equation within the context of three-dimensional magnetic merging is given by Craig \& Fabling (1998; see also Tassi et al. 2005). For the present our aim is simply to emphasize the changing character of the solution as the inflow strength decreases, that is, as $\alpha$ is reduced below $\beta$.

The characteristics of Equation (9) are given by $s \pm \beta \tau$ which correspond in the original variables to $\ln x+(\alpha+\beta) t$ and $\ln x+(\alpha-\beta) t$. The characteristics define the propagation of information and both are inward pointing and converging for $\alpha>\beta$. Thus, if a wave envelope located in the outer field is represented initially as the superposition of two pulses, one pulse will localize toward the origin on the fast timescale $(\alpha+\beta)^{-1}$, while the other will localize more slowly on the timescale $(\alpha-\beta)^{-1}$. It follows that the case $\alpha>\beta$ corresponds to growing field envelopes that steepen significantly, generating large currents close to $x=0$. Formally, this behavior corresponds to the formation of a current singularity at the origin. For $\alpha<\beta$, however, the characteristics of the slow pulse are outward pointing and diverging. This pulse therefore produces relatively weak currents as it propagates outward.

The singular behavior at the origin can be resolved only by non-ideal resistive effects. The resulting diffusive length scale determines the reconnection rate of the solution. This point is illustrated by the well-known, steady resistive solution described below.

\subsection{Singularity Resolution by Resistivity}

For an inviscid plasma $v=0$, the steady momentum equation (6) yields

$$
u=-\frac{\beta}{\alpha} b(x)+\gamma x
$$

where $\gamma$ is a constant. The steady induction equation (7) is now integrated to give

$$
b(x)=\frac{E}{\eta \mu} \operatorname{daw}_{ \pm}(\mu x)-\frac{\beta \gamma}{2 \eta \mu^{3}}\left(\mu x-\operatorname{daw}_{ \pm}(\mu x)\right) .
$$

Here, $E=$ const is the electric field component along the $z$ axis,

$$
\mu^{2}=\frac{\left|\alpha^{2}-\beta^{2}\right|}{2 \alpha \eta},
$$

and

$$
\operatorname{daw}_{ \pm}(x)=e^{\mp x^{2}} \int_{0}^{x} e^{ \pm s^{2}} d s
$$

is a Dawson function with the sign chosen to match that of $\alpha^{2}-\beta^{2}$. Expression (11) departs from the general steady solution only by the absence of an even component of the form 


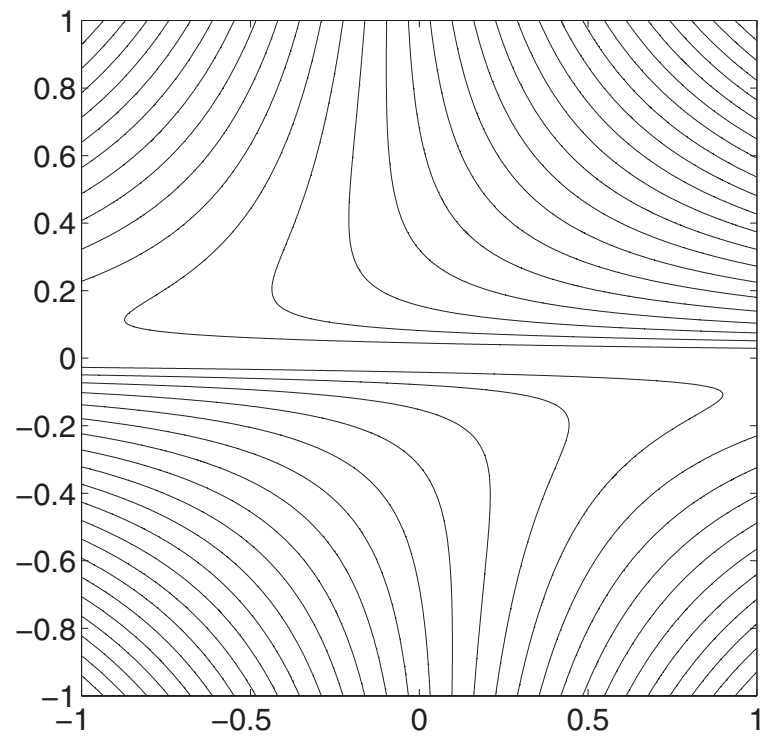

Figure 1. Magnetic field lines $\psi$ for inviscid reconnection in the case $\eta=10^{-3}$, $\alpha=1, \beta=0.25$, and $E=0.1$. Note the one-dimensional current layer overlying the origin.

$\exp \left(-\mu^{2} x^{2}\right)$ (Watson \& Craig 1997; Tassi et al. 2005) which gives rise to asymmetric current layers.

Solution (11) with $\alpha>\beta$ and $\gamma=0$ defines the reconnection model of Craig \& Henton (1995). In this case there is a welldefined current sheet at the origin of thickness

$$
x_{s} \simeq \sqrt{\frac{2 \eta}{\alpha^{-}}}, \quad \alpha^{-} \equiv \frac{\alpha^{2}-\beta^{2}}{\alpha} .
$$

The magnetic field achieves the strength

$$
b_{m} \simeq \frac{E}{\eta} x_{s} \simeq \frac{E}{\sqrt{\eta \alpha^{-}}}
$$

and falls off as $E /\left(\alpha^{-} x\right)$ outside the current layer. Clearly for physically relevant fields with $b_{m}=O(1)$ we require $E$ to scale as $\sqrt{\eta}$. The case $\beta=0$ recovers the annihilation model of Sonnerup \& Priest (1975). However, as the shearing parameter $\beta$ increases, the current layer broadens, becoming increasingly diffuse as $\beta \rightarrow \alpha$. The case $\beta>\alpha-$ not explored previously — provides a profile for $b(x)$ that grows exponentially with distance from the origin.

Finally, anticipating our numerical results, we emphasize the term involving $\gamma$. This allows an exact linear solution that lacks any small-scale structure:

$$
b=\frac{E}{\eta} x, \quad u=-\frac{\alpha}{\beta} b
$$

for $\gamma \beta=-2 E \mu^{2}$. More critically, this simple linear solution remains valid when the viscosity $v \neq 0$.

Figures 1 and 2 contrast the field lines of the Dawson function reconnection model with the uniform current solution of Equation (16). Notably, in Figure 2, the field accumulates at the outer boundary: there is no localized current layer overlying the origin.

\subsection{Singularity Resolution by Resistivity and Viscosity}

When both $\eta$ and $v$ are nonzero, the MHD system is too complicated to allow an exact analytical solution for reconnection of two-dimensional magnetic fields, although generalizations of the magnetic merging solution with $\beta=0$ are available

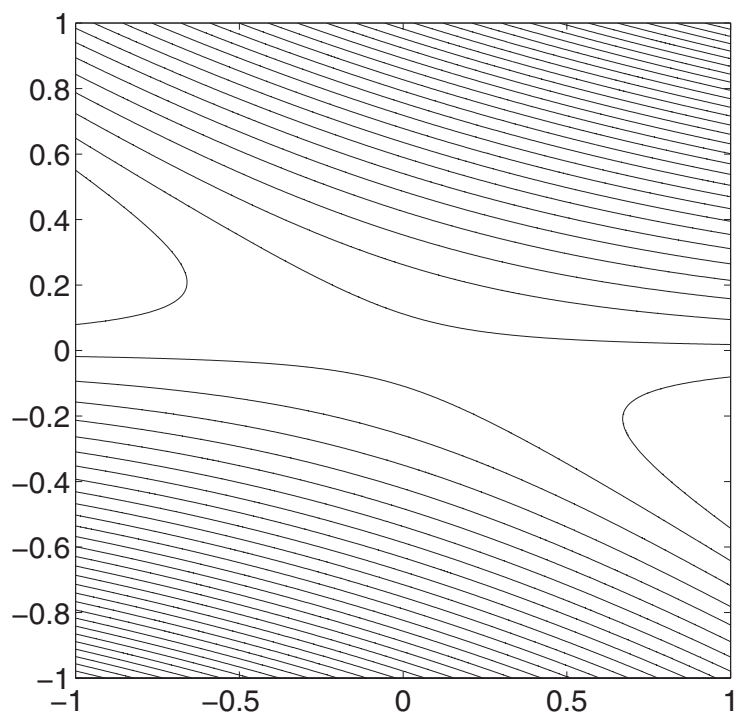

Figure 2. Sheared magnetic field $\psi$ for the case $\eta=10^{-3}, \alpha=0.25, \beta=1$, and $E=0.1$. In contrast to Figure 1 , this solution has a weak uniform current density.

(Sonnerup \& Priest 1975; Besser et al. 1990; Jardine et al. 1992). Notably, a visco-resistive length scale does not appear in any of those solutions.

To explore the structure of a more general steady reconnection solution in the physically interesting case $\eta \ll v \ll 1$, we assume that the daw $w_{+}$solution (11) approximates the magnetic field profile $b(x)$ everywhere except in a resistive current layer of thickness $\sqrt{\eta / \alpha^{-}}$. This is certainly the case in an ideal outer region where resistive and viscous effects are negligible. Substituting $b=E /\left(\alpha^{-} x\right)$ in the steady momentum Equation (6) yields

$$
\frac{v}{\alpha} u^{\prime \prime}+x u^{\prime}-u=\frac{2 \beta E}{\alpha \alpha^{-} x}, \quad x>\sqrt{\frac{\eta}{\alpha^{-}}} .
$$

The odd solution of this equation is

$$
u(\xi)=A \xi+\epsilon \xi \ln \xi-\frac{1}{6} \epsilon \xi^{3}{ }_{2} F_{2}\left(1,1 ; 2, \frac{5}{2} ;-\frac{1}{2} \xi^{2}\right),
$$

where ${ }_{2} F_{2}$ is a generalized hypergeometric function (e.g., Olver et al. 2010), $\xi=(\alpha / \nu)^{1 / 2} x>0$ and $\epsilon=2 \beta E /\left[(\alpha \nu)^{1 / 2} \alpha^{-}\right]$. The constant $A$ is defined by the matching condition $u(\infty)=0$ that follows from the ideal outer solution

$$
u(\xi \gg 1) \approx-\frac{\epsilon}{2 \xi} .
$$

To obtain an explicit expression for $A$, we use the reduction formula

$$
{ }_{2} F_{2}(1, b ; 2, d ; z)=\left(\frac{d-1}{b-1}\right) \frac{1}{z}\left[{ }_{1} F_{1}(b-1, d-1 ; z)-1\right]
$$

and write

$$
\begin{aligned}
{ }_{2} F_{2}\left(1,1 ; 2, \frac{5}{2} ;-\frac{1}{2} \xi^{2}\right)= & \frac{3}{2\left(-\xi^{2} / 2\right)} \lim _{\delta \rightarrow 0} \frac{1}{\delta} \\
& \times\left[{ }_{1} F_{1}\left(\delta, \frac{3}{2} ;-\frac{1}{2} \xi^{2}\right)-1\right] .
\end{aligned}
$$

We evaluate the limit by expanding the right-hand side in a Taylor series and substitute the result into Equation (18). 
On using Equation (19), we get

$$
A=-\epsilon \lim _{\xi \rightarrow \infty}\left[\ln \xi+\sum_{k=1}^{\infty} \frac{(-1)^{k} \xi^{2 k}}{2 k(2 k+1) ! !}\right],
$$

which gives $A \approx-0.365 \epsilon$.

Of particular interest is $u(\xi)$ in the range $(\eta / v)^{1 / 2}<\xi<1$, which describes the velocity profile within the viscous boundary layer:

$$
u(x) \approx \frac{2 \beta E}{v \alpha^{-}} x\left[\ln \left(\sqrt{\frac{\alpha}{v}} x\right)-0.365\right] .
$$

We observe that a visco-resistive scale does not emerge in the approximate steady solution for magnetic reconnection in two dimensions, except possibly in a next iteration. Thus, our analysis suggests that separate viscous and resistive length scales - rather than a single visco-resistive scale-tend to develop in planar reconnection dynamics.

\section{RELAXATION SIMULATIONS OF THE VISCO-RESISTIVE SYSTEM}

\subsection{Visco-resistive Relaxation}

We now turn to the relaxation of the visco-resistive system toward a steady equilibrium solution. Of central concern is the role of viscosity in the relaxation.

Recall that the only small scale deriving from the steady resistive solution is $x_{s} \simeq \sqrt{\eta / \alpha^{-}}$(see Section 2.4). In the visco-resistive case though, dimensional considerations, based on Equations (6) and (7) in steady state, suggest that any small scale should satisfy

$$
\left|\alpha^{2}-\beta^{2}\right| x_{s}^{4}=\eta v+\alpha x_{s}^{2}|\eta-v| .
$$

Three potential scales emerge

$$
\sqrt{\frac{\eta}{\alpha^{-}}}, \quad \sqrt{\frac{v}{\alpha^{-}}}, \quad\left(\frac{\eta v}{\alpha \alpha^{-}}\right)^{1 / 4} .
$$

We retain $\alpha^{-}$in the denominator to account for the possibility that small scales may be lost as $\beta \rightarrow \alpha$. We use numerical relaxation simulations to determine which of these scales emerges in a specific reconnection model.

To simulate the problem it is convenient to use stream and flux function representations of the fields:

$$
u=-f_{x}(x, t), \quad b=-g_{x}(x, t) .
$$

For reconnection fields that are odd functions of $x$ the potentials satisfy

$$
\begin{gathered}
f_{t}-\alpha x f_{x}+2 \alpha f=\beta x g_{x}-2 \beta g+\nu f_{x x}, \\
g_{t}-\alpha x g_{x}=\eta g_{x x}+\beta x f_{x} .
\end{gathered}
$$

The steady-state equations can be recovered using the replacements $f(x, t) \rightarrow f(x), g(x, t) \rightarrow g(x)-E t$.

In practice, the relaxation is modeled by specifying the parameters $\alpha, \beta, \eta, v$ and by assuming a fixed value of $E$. This formulation allows the reconnection fields to adjust dynamically to external flux washed through the outer boundary. The boundary conditions at the origin now follow from the symmetry of the potentials, $f_{x}(0, t)=g_{x}(0, t)=0$. At the outer

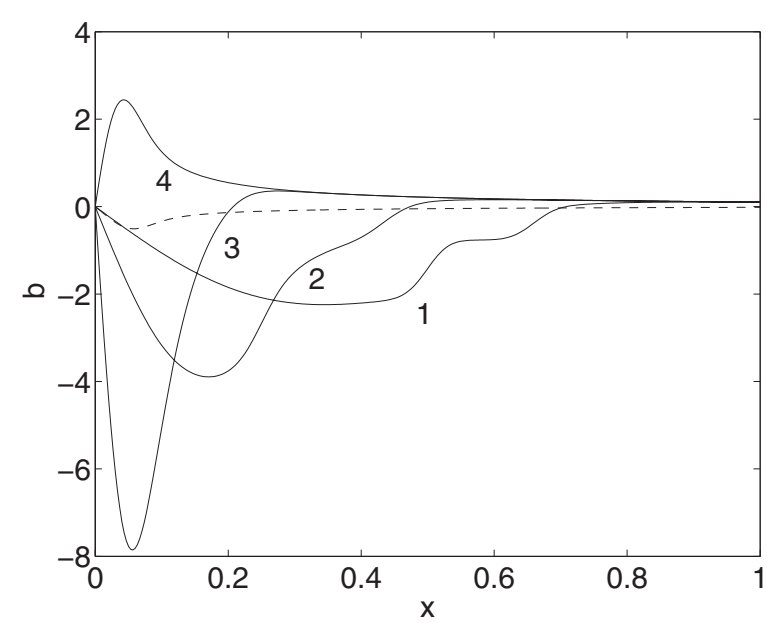

Figure 3. Inviscid relaxation of the reconnection magnetic field $b$ for the case $\eta=10^{-3}, \alpha=1, \beta=0.25$, and $E=0.1$. In the present run the relaxed velocity field (dotted line) closely approximates $u \simeq-\beta b / \alpha$. Times (1-4) correspond to $0.56,1.12,2.24$, and 10.0 Alfvén times.

boundary $x=1$ we take $g=0$ and extrapolate $f$ from the interior solution. The extrapolation means that the method cannot be used to find a solution with a boundary layer at $x=1$, which would be a visco-resistive analogue of the resistive solutions of daw_ type. This is not a significant limitation of the method, however, since we are primarily interested in reconnective solutions containing a localized current layer within the region, typified by the $\mathrm{daw}_{+}$resistive solution of Figure 1.

For example, in the simplest case of inviscid reconnection, a fully relaxed, well-resolved simulation should effectively "pick out" a unique member of the one parameter equilibrium family (11) specified by the value of $\gamma$. More generally, when analytic solutions are not available, the relaxation from arbitrary initial conditions should provide well-defined visco-resistive equilibria. In what follows the initial conditions are taken as

$$
g(x, 0)=x^{2}-1, \quad f(x, 0)=1+\cos (\pi x),
$$

consistent with smooth large-scale fields at $t=0$.

\subsection{The Visco-resistive System for $\alpha>\beta$}

Figure 3 shows the development of a localized current layer for a typical inviscid computation $\left(\alpha=1, \beta=0.25, \eta=10^{-3}\right.$, $v=0, E=0.1$ ). By around 10 Alfvén times the fields have relaxed to their equilibrium values. The resultant equilibria are found to closely approximate the Dawson function model with $u=-\beta b / \alpha, b=E$ daw $_{+}(\mu x) /(\eta \mu)$, and $\gamma=0$.

The relaxation can be clarified by introducing the auxiliary function

$$
h=f(x, t)+\frac{\beta}{\alpha} g(x, t) .
$$

Then the $h$-field must become small for large times if an inviscid solution with $f \simeq-\beta g / \alpha$ is to be achieved. The system becomes

$$
\begin{aligned}
h_{t}-\alpha^{+} x h_{x}+2 \alpha h-v h_{x x} & =\frac{\beta}{\alpha}\left(\alpha^{-} x g_{x}+(\eta-v) g_{x x}\right), \\
g_{t}-\alpha^{-} x g_{x} & =\eta g_{x x}+\beta x h_{x},
\end{aligned}
$$

where

$$
\alpha^{ \pm}=\frac{\alpha^{2} \pm \beta^{2}}{\alpha}
$$




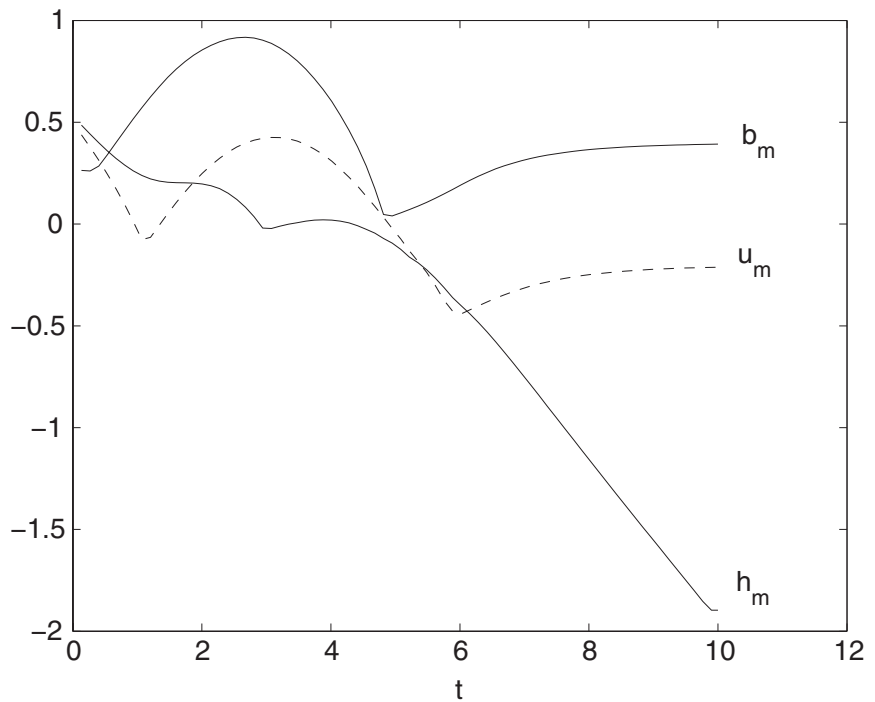

Figure 4. Comparison of field amplitudes for inviscid relaxation. The log-ten plot shows that, after the initial localization phase $0 \leqslant t \leqslant 5$, the $h$-field declines exponentially before flattening out for $t>10$. Parameters are those of Figure 3 .

A key property of this system is that for $v=0$ the $h$-field can evolve on a much faster timescale than the $g$-field. That is, since the initial gradients in $g$ are small (by virtue of the initial conditions), the right-hand side of Equation (31) is negligible for $\beta$ sufficiently small. Under these conditions $h$ localizes and decays rapidly according to

$$
h=h_{0}\left(x e^{\alpha^{+} t}\right) e^{-2 \alpha t},
$$

where $h_{0}$ is the initial profile. Since the $h$-wave establishes the reconnection fields at the level $f \simeq-\beta g / \alpha$, later times $t \gg 1 / \alpha^{+}$are characterized by the relatively slow relaxation

$$
g_{t}-\alpha^{-} g_{x}=\eta g_{x x} .
$$

This behavior is illustrated in Figure 4 which shows the log-ten amplitudes of the $b-, u-$, and $h$-fields. Following the initial phase ( $0 \leqslant t \leqslant 5$ for the present parameters), the $h$-field declines in amplitude while continuing to localize. However, it should not be assumed that the $h$-field vanishes completely even for large times (say $t>10$ ). This reflects the fact that minor deviations from the Dawson function model with $u=-\beta b / \alpha$ are present due to small but non-vanishing $\gamma$ (see Equation (10)).

Consider now the more physically interesting case $v \gg \eta$. As Figure 5 with $v=10 \eta$ shows, the equilibrium velocity field is strongly suppressed within the current layer $\left(x<x_{s} \simeq\right.$ $\left.\sqrt{\eta / \alpha^{-}}\right)$. Thus, it is only in the outer field that the inviscid relation $u \simeq-\beta b / \alpha$ holds.

To understand this behavior note that, even with $v \gg \eta$, there is still a fast initial phase in which the $h$-field rapidly localizes and decays. This decay is more pronounced than previously due to the significant viscous damping of the $h$-field. For longer times $\left(t \gg 1 / \alpha^{+}\right)$, however, the $h$-field can no longer decline because the viscous term on the right-hand side of Equation (31) provides an effective source term. It follows that in the high current region close to the origin where $v g_{x x}$ becomes large, the $b$-field induces growth in the $h$-field, effectively suppressing the reconnection velocity field $u(x, t)$ over the scale of the current layer.

The localization and induction phases are shown in Figure 6 for the case $v=10 \eta$. These profiles differ markedly from the

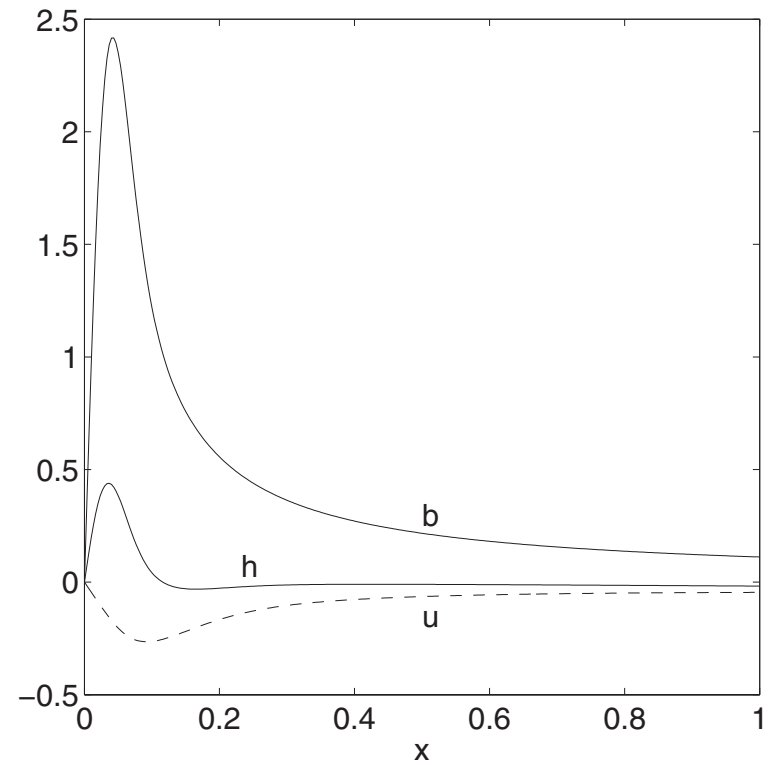

Figure 5. Profiles of the relaxed reconnection fields. Although the magnetic field (top plot) peaks on the resistive $\sqrt{\eta}$ scale, the velocity field (dotted line) is suppressed within the current layer and peaks on the larger viscous scale $\sqrt{v}$. The intermediate plot shows the $h$-field profile. Parameters are $\eta=10^{-3}$, $v=10^{-2}, \alpha=1, \beta=0.25$, and $E=0.1$.

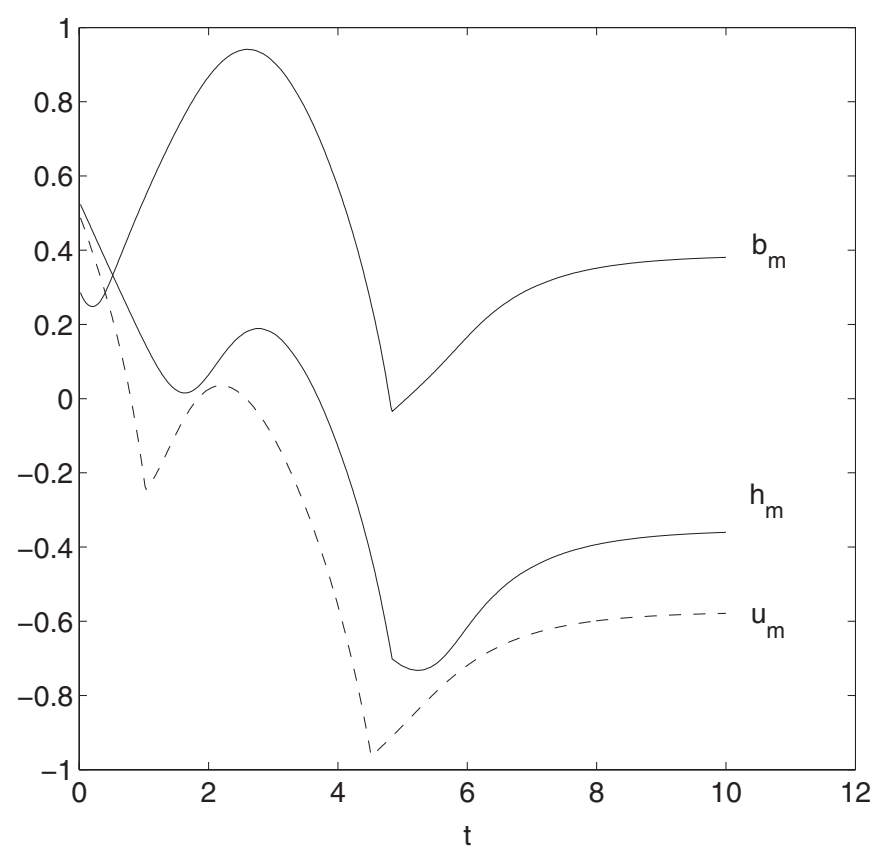

Figure 6. Comparison of (log-ten) field amplitudes in viscous relaxation. The localization phase for $t \leqslant 5$ is again evident, but in marked contrast to the ideal relaxation of Figure 4, the $h$-field, being induced by the strongly localizing $b$-field at the origin, is no longer small at large times. Parameters are those of Figure 5.

inviscid simulation of Figure 4. Clearly, in the case of strong viscosity, it is only in the outer field $x \geqslant \sqrt{v / \alpha^{-}}$that the reconnection velocity field is not strongly suppressed.

How are the peak magnetic and velocity fields related? If we assume that the magnetic and velocity field amplitudes are determined by the resistive and viscous scales, respectively, then for $v>\eta$ we obtain

$$
u_{m} \simeq \frac{\beta}{\alpha} \sqrt{\frac{\eta}{v}} b_{m}, \quad b_{m} \simeq \frac{E}{\sqrt{\eta \alpha^{-}}} .
$$




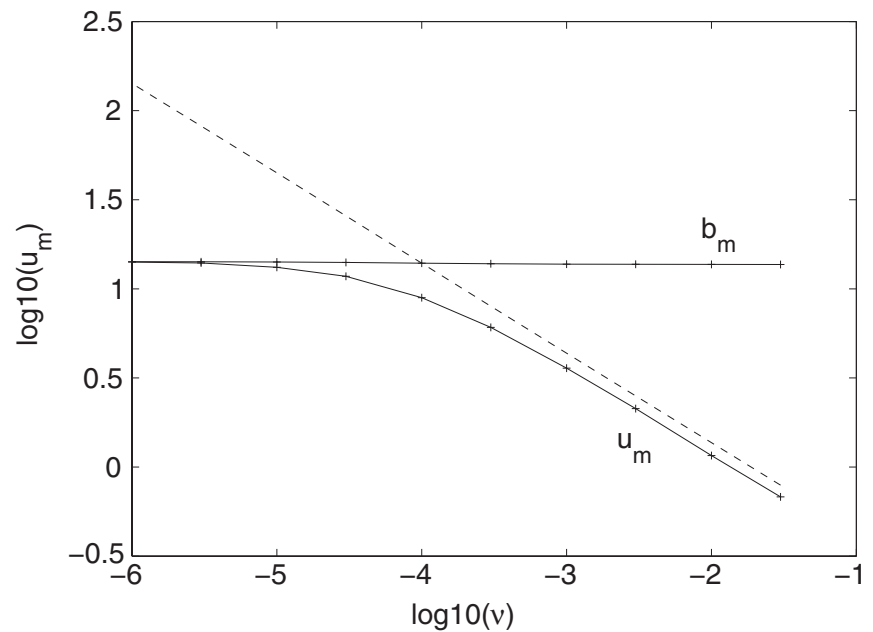

Figure 7. Scaling of the peak velocity and magnetic field with viscosity at fixed $\eta=10^{-4}$. The velocity field amplitude $u_{m}$ declines from the level $\beta b_{m} / \alpha$ as $v$ increases beyond $\eta$. Shown also is the reference line that derives from Equation (36). Note that for the purposes of visual comparison the velocity field has been scaled by the factor $\alpha / \beta$. Parameters are $\alpha=1, \beta=0.25$, and $E=0.1$.

Note that the approximate analytic solution (23) leads to $u_{m}=1.06 \beta E /\left[(\alpha \nu)^{1 / 2} \alpha^{-}\right]$. This is consistent with estimate (36) because a localized current layer emerges only if $\beta<\alpha$ and thus $\alpha^{-} \approx \alpha$. The veracity of the argument is further supported by Figure 7 which shows the scaling of the field amplitudes predicted by Equation (36). Here $\eta=10^{-4}$ is fixed and the viscosity is systematically varied between $10^{-6}$ and $10^{-1.5}$. We see that the velocity amplitude (scaled by the factor $\alpha / \beta$ to aid visual comparison) falls off as $v^{-1 / 2}$ in the case $v>\eta$.

\subsection{The Visco-resistive System for $\alpha \leqslant \beta$}

According to the analysis of the ideal system in Section 2, the system is expected to change character if $\alpha<\beta$. Relaxation computations indeed confirm that when the flow is no longer strong enough to localize the field, the field gets expelled from the merging region, accumulating flux in the vicinity of the outer boundary. Thus there is no reconnecting current layer. Even so, a severe pile-up of the field in the outer regions, as suggested by the resistive solution in terms of the Dawson function daw $-(\mu x)$, is not in evidence. What does occur is a relaxation to the linear solution (16).

Figure 8 shows time slices of a typical field relaxation for the parameters $\alpha=0.25, \beta=1, \eta=v=10^{-3}$, and $E=0.1$. The evolution contrasts sharply with the much faster relaxation of the flow-driven solution in Figure 3.

The form the relaxation takes can be explored by defining a discrepancy function $q$ as

$$
q=f(x, t)+\frac{\alpha}{\beta} g(x, t)
$$

The relaxation equations are now

$$
\begin{aligned}
q_{t}-2 \alpha x q_{x}+2 \alpha q & =\beta^{-}\left(x g_{x}-2 g\right)+\frac{\alpha}{\beta} \eta g_{x x}, \\
g_{t} & =\eta g_{x x}+\beta x q_{x},
\end{aligned}
$$

where

$$
\beta^{-}=\frac{\beta^{2}-\alpha^{2}}{\beta} \text {. }
$$

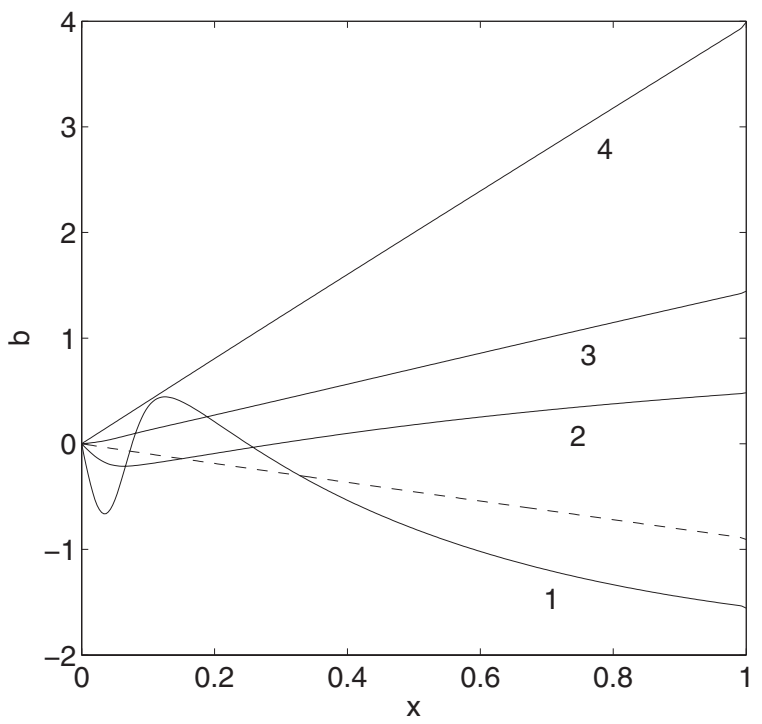

Figure 8. Relaxation of the reconnection magnetic field $b$ for the case $\alpha=0.25$, $\beta=1, E=0.1$, and $\eta=v=10^{-3}$. Profiles $1-4$ are snapshots taken at $2.2,11$, 17 , and 30 Alfvén times, respectively. The dotted line shows the velocity field at the final time $t \simeq 30$. The $b$-field amplitude achieves the level $E / \eta=100$ for sufficiently long times.

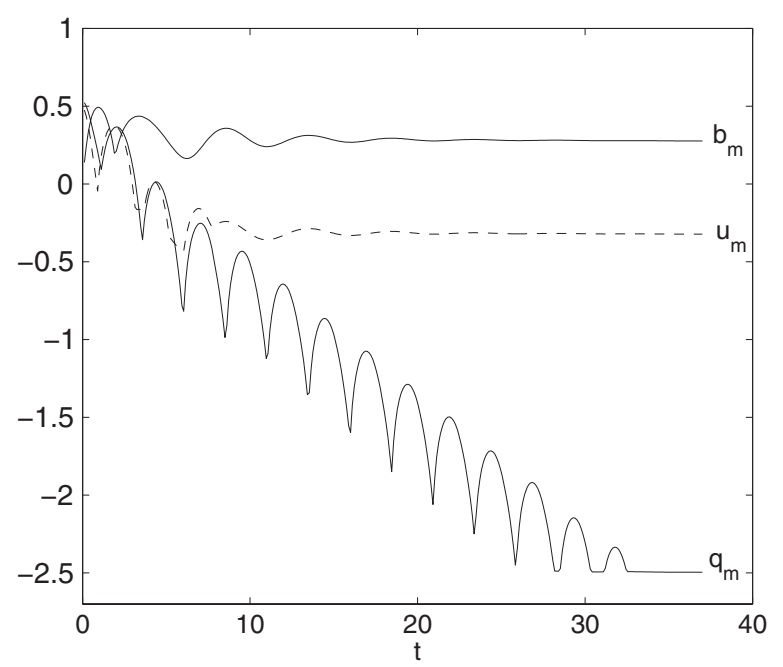

Figure 9. log-ten amplitudes of the reconnecting fields vs. time. The parameters are $\alpha=0.25, \beta=1$, and $\eta=v=E=10^{-3}$. Unlike the flow-driven reconnection solution of Figure 4, the discrepancy field $q$ is characterized by well-defined oscillations.

A key aspect of this system is that the induction Equation (39) is diffusive rather than advective in character. Accordingly, the late relaxation in which

$$
g(x) \rightarrow-\frac{E}{\eta} \frac{x^{2}}{2}, \quad f(x) \rightarrow-\frac{\alpha}{\beta} g(x),
$$

and $q \rightarrow 0$, proceeds very slowly, over a timescale determined by $\eta^{-1}$.

Computations show that the $q$-field has an oscillatory decay in contrast to the monotonic decline of the $h$-field in the flow-driven reconnection model. In Figure 9, these oscillations are shown for the parameters $v=\eta=E=10^{-3}$. For this calculation we have purposely assumed a relatively small value of $E$ : this ensures that the late evolution is dominated by oscillatory modes, as opposed to the growing field gradients of Figure 8. Independent of these considerations, increasing the viscosity always has the effect of damping out the oscillations more rapidly. 
The oscillation frequency of the $q$-field is accurately described by $\omega \simeq \sqrt{\beta^{2}-\alpha^{2}}$, which means that a Fourier mode $\exp (i k s+$ $i \omega t$ ) in the Klein-Gordon Equation (9) is characterized by a small value of $k$. An exact analytical calculation confirms that the equilibrium is approached via weakly damped Alfvénic oscillations of frequency $\omega=\beta$ and decay rate $\sim|\ln \eta|^{-3}$ when $v=\alpha=0$ (Bulanov et al. 1990). However, the interpretation of the decay rate is more subtle since oscillatory wave modes have the freedom to carry energy out of the source volume (Craig \& Fabling 1998).

\subsection{Summary}

The present relaxation solutions fall into two distinct categories, depending on whether or not the merging is flow dominated. Neither category provides evidence of a visco-restive scale. Thus, in the case $\alpha>\beta$, the system is characterized by the emergence of a localized, reconnective current layer of thickness $\sim \sqrt{\eta}$. In the absence of viscosity the reconnection fields in the case $\alpha>\beta$ satisfy $u(x) \simeq-\beta b(x) / \alpha$, where $b(x)$ is well approximated by the Dawson function model, i.e., Equation (11) with $\gamma \simeq 0$. Realistic levels of viscosity have the effect of suppressing the velocity field within the current layer and, for $v \gg \eta$, the peak in $u(x)$ is shifted to the broader viscous scale $\sim \sqrt{\nu}$. The reconnection rate, however, remains independent of $v$. More specifically, since the steady reconnection field $b(x)$ is always controlled by the resistive scale $x_{s} \sim \sqrt{\eta}$, flow-dominated merging rates remain well approximated by the resistive Dawson function model even in the limit $v \gg \eta$. These conclusions are in good agreement with the approximate analytical solution of Section 2.5 for steady visco-resistive reconnection in two dimensions.

The geometry of the emerging steady solution changes radically when the magnetic field dominates the imposed flow $(\alpha<\beta)$. Localized current layers are no longer achieved and what emerges from the dynamic relaxation are the simple linear solutions of Equation (16), namely $b(x)=E x / \eta$, $u(x)=-\alpha b(x) / \beta$. The more extreme equilibria associated with the daw $\_(\mu x)$ functions are not achieved. Unlike the flow-driven solutions, the linear equilibria are exact solutions, valid for any value of $v$. The relaxation now involves damped oscillatory Alfvén modes whose frequency is given by $\sqrt{\beta^{2}-\alpha^{2}}$. Although the dynamic interaction of these waves with the outer boundary has the potential to provide transient visco-resistive layers of thickness $\sim(\eta v)^{1 / 4}$, these layers are conspicuously lacking in the equilibrium solutions themselves.

\section{DISCUSSION}

We have used relaxation simulations to obtain steady magnetic reconnection solutions of a visco-resistive MHD system. Our main finding is that, in contrast to closed line-tied $X$-points (Craig et al. 2005), the visco-resistive scale $(\eta v)^{1 / 4}$ does not emerge in flux pile-up magnetic reconnection in open geometry. This result is significant because a fluid model based on the visco-resistive scale would lead to a very slow ohmic energy release rate, a factor of $(\eta / v)^{1 / 4}$ slower than the resistive Sweet-Parker rate. Moreover, as long as the large-scale reconnection inflow is fast enough $(\alpha>\beta)$, the resistive solution defined by the Dawson function model provides a good approximation to the magnetic field profile even for $v \gg \eta$. Hence our results imply that the scaling arguments for the parameters of the Sweet-Parker current sheet in the case $v \gg \eta$ (Park et al. 1983) are not universally valid (see also Armstrong et al. 2011). More generally, the $\eta^{1 / 2}$ scaling obtained in our visco-resistive simulations implies that a complete description of a flaring current sheet would need to incorporate collisionless effects.

An important aspect of our approach is that the boundary velocity and magnetic fields are free to adjust to the internal dynamics of the relaxation. Although this approach is useful for investigating how ideal singularities within a volume are resolved by resistive and viscous effects in internal boundary layers, it is possible that other strategies-say, fixing the reconnecting fields at the outer boundary-could lead to seemingly conflicting results. Litvinenko (2006) takes $u(1, t)=b(1, t)=1$ for the system with $\alpha=0, \beta=1$ and shows that both the flow vorticity and the electric current density are localized on a scale of order $(\eta \nu)^{1 / 4}$ at the outer boundary rather than elsewhere in the region. By contrast, the present analysis provides steady solutions either without a small-scale structure (if $\alpha \leqslant \beta$ ) or with separate viscous and resistive layers within the merging region (if $\alpha>\beta$ ). To reconcile these results it should be remembered that the present computation deals with open geometries in which boundary fields can adjust dynamically. Thus, while outward propagating waves impacting the outer boundary can (and do) provide localized transient effects (especially in the case $\alpha<\beta$ ), these rapidly disperse during the relaxation allowing smooth, global equilibria to develop.

It is interesting to compare these findings with the study by Tassi et al. (2005). Tassi et al. (2005) investigated whether the exact, steady resistive solutions of Craig \& Henton (1995) could be reached by a dynamically evolving MHD system when disturbances of the velocity shear and magnetic field are superimposed onto initial large-scale fields containing a null point. A natural question is whether the analysis of Tassi et al. (2005), based on the field Equations (10) and (11) with prescribed boundary conditions, can be generalized to explore the dynamic accessibility of visco-resistive reconnection solutions. This is made difficult because the reconnection solution of Craig \& Henton (1995) cannot be extended to include viscosity unless either the magnetic field or plasma flow is strictly one-dimensional (e.g., Besser et al. 1990; Litvinenko 2006). Hence in this paper we have adopted a more restricted approach: we obtain steady viscoresistive solutions using relaxation simulations and explore how these solutions deviate from the resistive reconnection models as the viscosity is systematically increased. Once the relaxation is achieved, however, it is straightforward to test the resulting equilibria for dynamic accessibility. A preliminary analysis suggests that the visco-resistive solutions of this paper are in fact dynamically accessible.

This work was partially supported by NASA through a grant to the University of New Hampshire. Useful suggestions by an anonymous referee are acknowledged.

\section{REFERENCES}

Armstrong, C. K., Craig, I. J. D., \& Litvinenko, Y. E. 2011, A\&A, 534, A25 Besser, B. P., Biernat, H. K., \& Rijnbeek, R. P. 1990, Planet. Space Sci., 38, 411

Biskamp, D. 1994, Phys. Rep., 237, 179

Braginskii, S. I. 1965, Rev. Plasma Phys., 1, 205

Bulanov, S. V., Shasharina, S. G., \& Pegoraro, F. 1990, Plasma Phys. Control. Fusion, 32, 377

Cassak, P. A., Drake, J. F., \& Shay, M. A. 2006, ApJ, 644, L145

Craig, I. J. D., \& Fabling, R. B. 1998, Phys. Plasmas, 5, 635

Craig, I. J. D., \& Henton, S. M. 1995, ApJ, 450, 280

Craig, I. J. D., \& Litvinenko, Y. E. 2005, Phys. Plasmas, 12, 112105

Craig, I. J. D., Litvinenko, Y. E., \& Senanayake, T. 2005, A\&A, 433, 1139

Fabling, R. B., \& Craig, I. J. D. 1996, Phys. Plasmas, 3, 2243 
Hollweg, J. V. 1986, ApJ, 306, 730

Jardine, M., Allen, H. R., Grundy, R. E., \& Priest, E. R. 1992, J. Geophys. Res., 97, 4199

Litvinenko, Y. E. 2005, Sol. Phys., 229, 203

Litvinenko, Y. E. 2006, Phys. Plasmas, 13, 092305

Litvinenko, Y. E. 2009, ApJ, 694, 1464

Malakit, K., Cassak, P. A., Shay, M. A., \& Drake, J. F. 2009, Geophys. Res. Lett., 36, L07107
Olver, F. W. J., Lozier, D. W., Boisvert, R. F., \& Clark, C. W. 2010, NIST Handbook of Mathematical Functions (Cambridge: Cambridge Univ. Press), Chap. 16

Park, W., Monticello, D. A., \& White, R. B. 1983, Phys. Fluids, 27, 137

Sonnerup, B. U. Ö., \& Priest, E. R. 1975, J. Plasma Phys., 14, 283

Tassi, E., Titov, V. S., \& Hornig, G. 2005, Phys. Plasmas, 12, 112902

Titov, V. S., \& Priest, E. R. 1997, J. Fluid Mech., 348, 327

Watson, P. G., \& Craig, I. J. D. 1997, Phys. Plasmas, 4, 101 